\title{
Sulforaphane Does Not Protect Right Ventricular Systolic and Diastolic Functions in Nrf2 Knockout Pulmonary Artery Hypertension Mice
}

\author{
Guangyan Zhang ${ }^{1,2,3} \cdot$ Yin Kang $^{1,2,3} \cdot$ Dakotah Cathey $^{1} \cdot$ Amanda J. LeBlanc $^{4} \cdot$ Jun Cai $^{3,5} \cdot$ Lu Cai $^{3,5} \cdot$ Sheng Wang $^{2,6}$. \\ Jiapeng Huang ${ }^{1,4,5,7}$ (D) Bradley B. Keller ${ }^{5,8,9}$
}

Accepted: 2 February 2022 / Published online: 14 February 2022

(c) The Author(s), under exclusive licence to Springer Science+Business Media, LLC, part of Springer Nature 2022

\begin{abstract}
Purpose Nrf2 is a nuclear transcription factor and plays an important role in the regulation of oxidative stress and inflammation. We recently demonstrated that sulforaphane (SFN) protected mice from developing pulmonary arterial hypertension $(\mathrm{PAH})$ and right ventricular (RV) dysfunction by elevating cardiac Nrf2 expression and function. Here we further investigate Nrf2 dependence for SFN-mediated prevention of PAH and RV dysfunction in an Nrf2 knockout mouse model.

Methods We used male global Nrf2-knockout mice and male C57/6 J wild type mice in the following groups: Control group received room air and vehicle control; SuHx group received SU5416 and 10\% hypoxia for 4 weeks to induce PAH; $\mathrm{SuHx}+\mathrm{SFN}$ group received both SuHx and sulforaphane, a Nrf2 activator, for 4 weeks. Transthoracic echocardiography was performed to quantify RV function and estimate pulmonary vascular resistance over 4 weeks. PAH was confirmed using invasive RV systolic pressure measurement at 4 weeks.

Results All Nrf2 knockout mice survived the 4-week SuHx induction of PAH. SuHx caused progressive RV diastolic/systolic dysfunction and increased RV systolic pressure. The development of RV diastolic dysfunction occurred earlier in the Nrf2 knockout PAH mice when compared with the wide type PAH mice. SFN partially or completely reversed SuHx-induced RV diastolic/systolic dysfunction and increased RV systolic pressure in wild-type mice, but not in Nrf2 knockout mice.

Conclusion Our findings demonstrated the essential role of Nrf2 in SFN-mediated prevention of RV dysfunction and PAH, and increasing Nrf2 activity in patients with PAH may have therapeutic potential.
\end{abstract}

Keywords Pulmonary hypertension $\cdot$ Right heart $\cdot$ Right ventricle $\cdot$ Sulforaphane $\cdot$ Inflammation $\cdot \mathrm{Nrf} 2$

Guangyan Zhang, Yin Kang, Sheng Wang, Jiapeng Huang, and Bradley B. Keller contributed equally to this work.

Jiapeng Huang

j0huan03@louisville.edu

Sheng Wang

shengwang_gz@163.com

Bradley B. Keller

brad.keller@cchmc.org

1 Department of Anesthesiology and Perioperative Medicine, University of Louisville, 530 South Jackson Street, Louisville, KY, USA

2 Department of Anesthesiology, Guangdong Provincial People's Hospital, Guangdong Academy of Medical Sciences, Guangzhou, China

3 Pediatric Research Institute, Department of Pediatrics, University of Louisville, Louisville, KY, USA

4 Cardiovascular Innovation Institute, Department of Cardiovascular and Thoracic Surgery, University of Louisville, Louisville, KY, USA
5 Department of Pharmacology and Toxicology, University of Louisville, Louisville, KY, USA

6 Department of Anesthesiology, Guangdong Cardiovascular Institute, Guangdong Provincial People's Hospital,

Guangdong Academy of Medical Sciences, Guangzhou, China

7 Department of Medicine, University of Louisville, Louisville, KY, USA

8 Kosair Charities Pediatric Heart Research Program, Cardiovascular Innovation Institute, Department of Pediatrics, University of Louisville, School of Medicine, Louisville, KY, USA

9 Present Address: Cincinnati Children's Heart Institute, Greater Louisville and Western Kentucky Practice, Louisville, KY, USA 


\section{Introduction}

Pulmonary arterial hypertension (PAH) is a life-threatening disease characterized by progressive pulmonary vascular remodeling, chronically increased pulmonary arterial pressure, and right ventricular (RV) overload. RV failure eventually develops at the later stage and is the leading cause of death in PAH patients [1]. The median survival time for patients with PAH was 2.8 years [2], and the 5 -year survival rate was only $50 \%$ [3]. Life quality and survival in patients with PAH is closely related to RV function [4]. Currently, effective and targeted therapies for RV dysfunction are limited.

Inflammation has been established to play an important role in the pathogenesis of PAH, but the specific molecular mechanisms are largely unknown [5]. Lung tissues of PAH patients showed signs of chronic inflammation with increased circulating cytokine levels and perivascular inflammatory infiltrates [6, 7]. In the mouse monocrotaline-induced PAH and RV failure model, inflammation gradually worsens with the progression of the disease, including increased tumor necrosis factor- $\alpha$ (TNF- $\alpha)$ and interleukins [8]. Anti-inflammatory therapies such as epoprostenol, prostacyclin, and bosentan have been tested in animal models and clinical studies with success [9-11].

Nuclear factor erythroid 2-related factor (Nrf2) is a transcription factor that integrates cellular stress signals by directing various transcriptional programs. It plays a crucial role in anti-oxidative and anti-inflammatory responses in the cells. Accumulating evidence indicates that Nrf2 activation could be a novel target for treatment of inflammatory lung diseases [12-14]. In our previous study, we demonstrated that sulforaphane (SFN) prevented RV dysfunction and reduced pulmonary vascular remodeling in Su5416 and hypoxia exposure ( $\mathrm{SuHx}$ ) induced $\mathrm{PAH}$ mice. These effects were associated with up-regulation of $\mathrm{Nrf} 2 / \mathrm{NQO} 1$ and downregulation of inflammation and fibrosis [15]. However, it is unclear whether SFN mediated reversal of $\mathrm{RV}$ dysfunction and pulmonary vascular remodeling is dependent on the Nrf2 pathway. In this study, we aim to further investigate whether SFN could prevent PAH and RV dysfunction in an Nrf2 knockout mouse model. We elected to perform only echocardiographic evaluation and RV pressure measurements in this study without molecular and histological evaluations because if SFN does not protect RV dysfunction in Nrf2 knockout PAH mice, the likelihood of finding molecular and histological differences in Nrf2 knockout mice is very low.

\section{Methods}

\section{Animals}

Male C57/6 J wild type and Nrf2 global knockout mice at 7 weeks were purchased from Jackson lab (Bar Harbor, $\mathrm{ME})$. Nrf2 deficiency was confirmed by western blot in Nrf2 knockout (KO) mice. All mice were kept under the same conditions for one week before the study. All experimental procedures were approved by the Institutional Animal Care and Use Committee of the University of Louisville.

\section{PAH Induction}

PAH was induced by weekly subcutaneous (s.c.) injection of a VEGFR inhibitor (Su5416, $20 \mathrm{mg} / \mathrm{kg}$ body mass; Semaxinib, Adooq 5, USA), accompanied by 4 weeks of hypoxia (10\% oxygen) in commercially designed chambers (Oxycycler model A44XO, Biospherix, Redfield, NY). Control mice received only diluent and were housed in normoxic conditions within identical chambers. C57/6 J mice were randomly divided into three groups: Control (CTL: vehicle + normoxic air $n=4)$; SuHx (Su5416+ hypoxia exposure, $n=6)$; SuHx+SFN (Su5416+ hypoxia exposure + SFN injection, $n=6)$. Nrf2 knockout mice were also randomized into three groups: Control (CTL: vehicle + normoxic air $n=4)$; SuHx (Su5416 + hypoxia exposure, $n=4)$; SuHx + SFN (Su5416+ hypoxia exposure + SFN injection, $n=5$ ). SFN (Sigma-Aldrich, USA) was given at the dosage of $0.5 \mathrm{mg} / \mathrm{kg}$ s.c. for 5 days of each week based on our previous study [15]. We did not include SFN only groups in CTL and Nrf2 knockout mice because our previous study showed SFN alone had minimal effects on PAH and RV function [15]. We did not include SFN only control group because we have extensively studied and reported there was no impact of SFN on cardiac functions in Nrf2-KO mice. When we examined whether the Nrf2 pathway plays an essential role in SFN prevention of diabetes, angiotensin II, and intermittent hypoxia-induced cardiomyopathy (chronic mouse model with 3-month treatment of SFN), SFN had no effect on cardiac functions in Nrf2 KO mice [29-31]. Our earlier work also showed that SFN alone had no effects on cardiac functions in WT mice [15]. Therefore, we did not include SFN alone control groups in WT and Nrf2 KO mice for this study.

\section{Echocardiographic Measurements}

Two-dimensional and Doppler transthoracic echocardiography was performed with a high-frequency, high-resolution 
digital imaging platform (Vevo® 2100 Imaging System, FUJIFILM Visual Sonics Inc., Toronto, Canada). Echocardiography was performed by a trained individual (GZ) and the images were evaluated by a cardiologist (BBK) to ensure image quality and measurement accuracy, similar to our prior publication [15]. BBK was blinded to the grouping. Mice were anesthetized with $1 \%$ to $2 \%$ isoflurane and laid on their backs on a warming pad at $37^{\circ} \mathrm{C}$. Images were stored in the ultrasound system's hard drive for analysis.

\section{Invasive RV Pressure Recording}

At the end of 4 weeks before sacrifice, RV systolic pressure was recorded by a $1.2 \mathrm{~F}$ transducer-tipped pressure catheter (model FTH-1211B-0081, Transonic, London, ON, Canada). Mice were anesthetized with $1 \%$ to $2 \%$ isoflurane and laid on their backs on a warming pad as described before. Hair over the right jugular was removed using a depilatory gel, the skin was sterilized and tissues were dissected. The catheter was placed into the right jugular vein and advanced into the RV. Closed chest RV pressures ( $n>30$ waveforms per animal) were recorded during a steady state and averaged peak RV pressure and waveforms were further analyzed using a peak detection software (LabChart Pro Software, AD Instruments, Colorado Springs, CO). Right ventricular peak RV pressure rate of rise (dP/dt max), peak $\mathrm{RV}$ pressure rate of decline $(\mathrm{dP} /$ $\mathrm{dt}$ min) were calculated. $\mathrm{dP} / \mathrm{dt}_{\max }=$ slope of maximum derivative of change in systolic pressure over time; $\mathrm{dP} / \mathrm{dt}_{\min }=$ minimum derivative of change in diastolic pressure over time.

\section{Statistical Analysis}

Analysis was performed using Prism GraphPad 8 Software. All measurements were presented as mean \pm standard

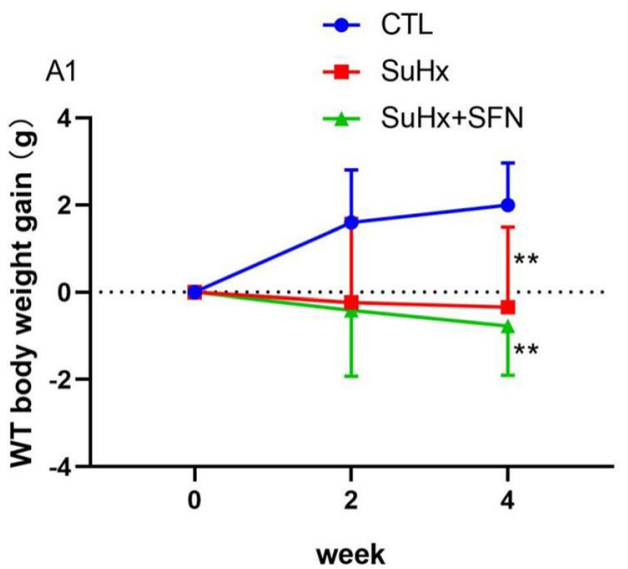

Fig. 1 Body weight changes during SuHx-induced PAH and SFN treatment. A1: wild type mice: body weight dropped significantly at week 4 in both SuHx and SuHx + SFN groups. CTL (blue, $n=4$ ), $\mathrm{SuHx}($ red, $n=6$ ), $\mathrm{SuHx}+\mathrm{SFN}$ (green, $\mathrm{n}=6$ ) groups, respectively. A2: deviation (SD). $P$ value $<0.05$ was considered statistically significant. Normality of data was checked and either logtransformation or non-parametric testing was performed on non-normally distributed data. Two-way ANOVA for repeated measurements followed by Tukey test was used for echocardiographic and body weight analysis during the time series. One-way ANOVA with Tukey comparison between groups was used for RV systolic pressure and heart rate analysis. Unpaired T-test was used to compare differences between wild type and Nrf2 knockout mice in terms of echocardiographic variables for $\mathrm{SuHx}$ to control and $\mathrm{SuHx}+\mathrm{SFN}$ to $\mathrm{SuHx}$ ratios.

\section{Results}

\section{Effects of SuHx and SFN on Body Weight in Wild Type and Nrf2 Knockout Mice}

All Nrf2 knockout mice survived the 4 weeks' hypoxic environment and Su5416 injection to induce PAH. Body weight showed similar increase over 4 weeks in both wild-type and Nrf2-knockout control mice. The body weight of SuHx and $\mathrm{SuHx}+\mathrm{SFN}$ groups (both wild type and Nrf2 knockout mice) decreased significantly at the fourth week. However, there was no significant difference in body weight between the wild type and Nrf2 knockout mice groups (Fig. 1).

\section{Effects of SuHx in Nrf2 Knockout Mice}

SuHx caused global RV systolic and diastolic dysfunction in both wild type and Nrf2 knockout mice. Cardiac index (CI) declined 2 weeks after SuHx treatment in Nrf2 knockout mice similar to wild type mice (Fig. 2, A2). Pulmonary valve

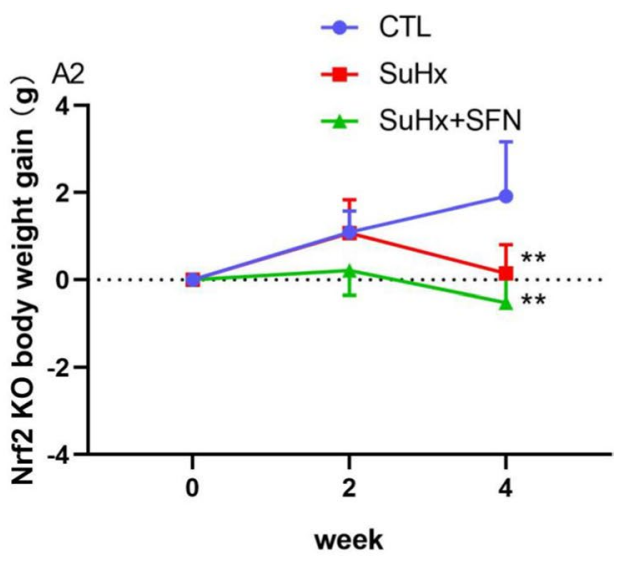

Nrf2 knockout mice: body weight dropped significantly at week 4 in SuHx and SuHx + SFN groups. CTL (blue, $n=4)$, SuHx (red, n=4), $\mathrm{SuHx}+\mathrm{SFN}$ (green, $n=5$ ) groups, respectively. ${ }^{*} * P<0.05$ versus CTL at week 4 

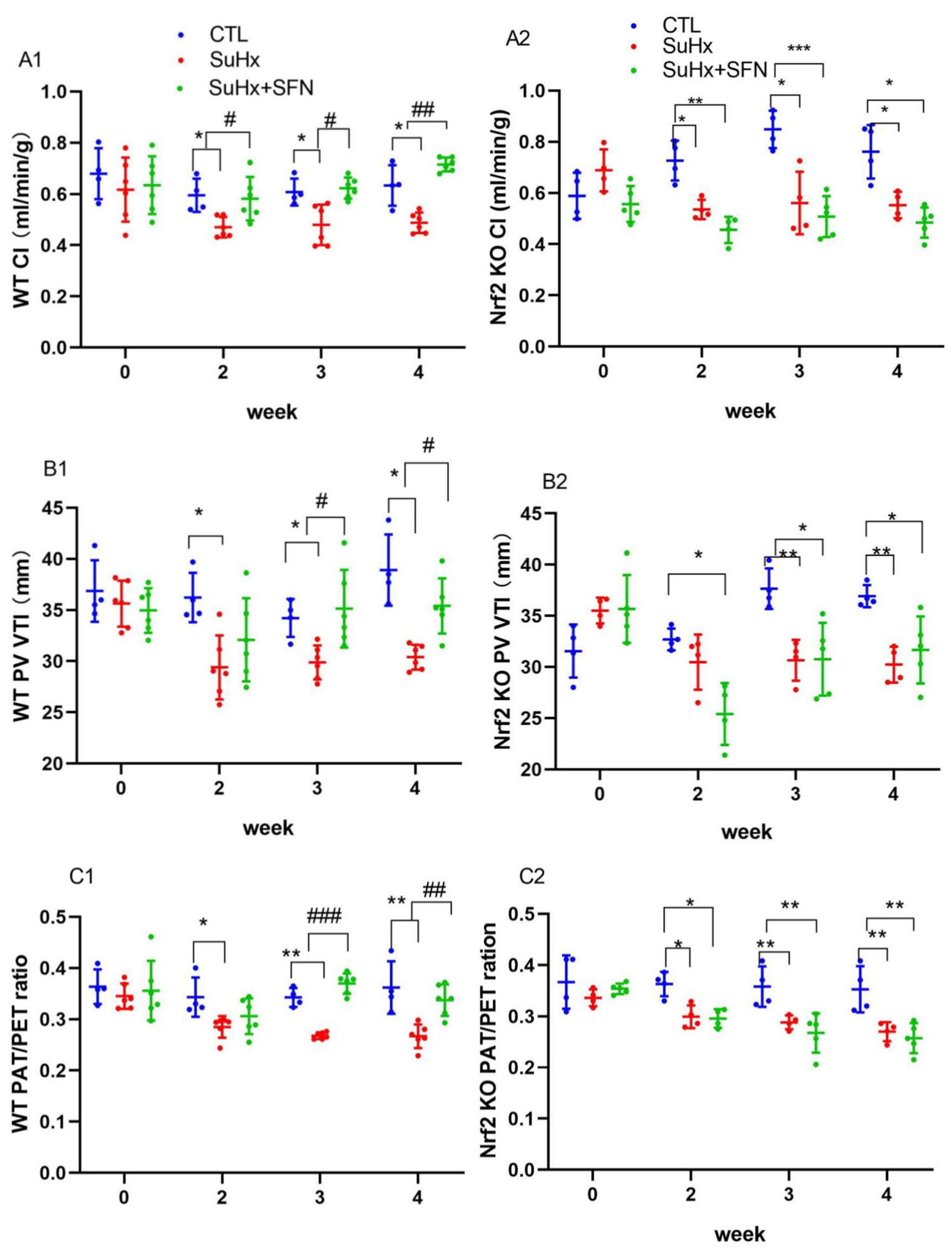
४Fig. 2 Reduced RV cardiac index (CI), pulmonary valve velocity time integral (PV VTI), and pulmonary artery acceleration/ejection time ratio (PAT/PET) by SuHx was attenuated by SFN in wild type mice, but not in Nrf2 knockout mice. A1: CI. B1: PV VTI. C1: PAT/PET ratio in wild type mice. $\mathrm{n}=4,6,6$, for CTL (blue), $\mathrm{SuHx}$ (red), and SuHx+SFN (green) groups, respectively. A2: CI. B2: PV VTI. C2: PAT/PET ratio in Nrf2 knockout mice. $\mathrm{n}=4,4,5$, for CTL (blue), SuHx (red), and SuHx+SFN (green) groups, respectively. $* P<0.05$, $* * P<0.01, \quad * * * P<0.001, \quad \# P<0.05, \quad \# \# P<0.01, \quad \# \# \# P<0.001$ between indicated groups at the same time point

velocity time integral (PV VTI), an index of stroke volume from the right ventricle, and pulmonary artery acceleration/ ejection time ratio (PAT/PET), an indicator of pulmonary hemodynamics and pulmonary vascular resistance, deteriorated at week 2 and persisted into week 4 after SuHx treatment in Nrf2 knockout mice similar to wild type mice (Fig. 2, B2 and C; Fig. 5A). Tricuspid annular plane systolic excursion (TAPSE), an indicator of RV systolic function, showed significant deterioration at week 2 and persisted into week 4 after SuHx treatment in Nrf2 knockout mice similar to wild type mice (Fig. 3, A2; Fig. 5A). Interestingly, RV E/E', an indicator of RV diastolic dysfunction, showed deterioration starting from week 2 and persisted into week 4 in Nrf2 knockout $\mathrm{SuHx}$ mice in contrast to the wild type SuHx mice, which demonstrated dysfunction starting from week 3 (Fig. 3, B2). Anatomically, RV free wall thickness (RVFW), an indicator of chronic RV hypertrophy to adapt to pressure overload in PAH, revealed significant increase since week 2 and persisted into week 4 in Nrf2 knockout SuHx mice similar to wild type SuHx mice (Fig. 3, C2). RV Tei index, a global assessment of both systolic and diastolic RV function, increased significantly in SuHx mice since week 3 and persisted into week 4 when compared to control mice (Fig. 3, D2).

\section{Effects of SFN in Wild Type and Nrf2 Knockout PAH Mice on Echocardiographic Parameters}

In wild type PAH mice, SFN attenuated the changes of CI, PV VTI, PAT/PET ratio, TAPSE, and RVFW thickness at week 2, 3, 4 (Fig. 2, A1, B1, C1; Fig. 3, A1, C1), and changes of RV E/E', RV Tei index at week 3, 4 (Fig. 3, B1, D1).

However, in Nrf2 knockout PAH mice, SFN did not reverse CI, PV VTI, PAT/PET ratio, TAPSE, RV E/E', RVFW thickness, and RV Tei index (Fig. 2, A2, B2, C2; Fig. 3, A2, B2, C2, D2).

\section{SFN Did Not Reverse Invasively Measured RV Systolic Pressure and Derivatives in Nrf2 Knockout Mice}

At the end of week 4, we invasively measured the peak RV systolic pressure (RVSP), peak RV pressure rate of rise
(dP/dT max), and peak rate of RV pressure decline (dP/dT $\mathrm{min}$ ) in mice. Heart rates showed no difference between any groups (Fig. 4, C1, C2). RVSP is a surrogate for pulmonary pressure and a measurement of the severity of PAH and $\mathrm{dP} /$ dT max is an important parameter of RV systolic function. Both RVSP and dP/dT max were significantly increased in both wild type and Nrf2 knockout SuHx mice (Fig. 4, A1, B1; A2, B2; Fig. 5B). The time course of pressure fall during isovolumic relaxation provides an important measure of early diastolic performance and can be described by the peak rate of pressure decline $\mathrm{dP} / \mathrm{dT} \min [32,33]$. $\mathrm{dP} / \mathrm{dT}$ min of SuHx wild type mice were significantly higher (more negative) than control mice, while SuHx Nrf2 knockout mice showed numerically higher (more negative) $\mathrm{dP} / \mathrm{dT}$ min, yet without statistical significance (Fig. 4, D1, D2; Fig. 5B).

SFN reversed the elevated RVSP and dP/dT max in wild type SuHx mice; however, SFN did not decrease either the RVSP or dP/dT max in Nrf2 knockout SuHx mice (Fig. 4, $\mathrm{A} 1, \mathrm{~A} 2$; B1, B2). SFN was able to restore $\mathrm{dP} / \mathrm{dT}$ min to the level of control in wild type SuHx mice (Fig. 4, D1), but SFN showed no significant $\mathrm{dP} / \mathrm{dT}$ min reversal in Nrf2 knockout SuHx mice (Fig. 4, D2) (Fig. 5).

\section{Comparison between WT and Nrf2 KO Mice Under Effects of SuHx and SFN}

SuHx affected WT and Nrf2 mice similarly for all echocardiographic variables at weeks 2, 3, and 4 (Tables 1, 2, and $3)$. However, at week 2 , SFN showed less protection for CI in Nrf2 KO SuHx mice when compared to WT SuHx mice (Table 1). At week 3, SFN showed less protection for CI, PV VTI, PAT/PET, TAPSE, RVFW, and RV Tei index in Nrf2 KO SuHx mice when compared to WT SuHx mice (Table 2). At week 4, SFN showed less protection for CI, RV E/E', RVFW, and RV Tei index in Nrf2 KO SuHx mice when compared to WT SuHx mice (Table 3).

\section{Discussion}

Our study demonstrated that SuHx caused RV systolic dysfunction and increased RV systolic pressure in Nrf2 knockout mice similar to wild type mice. Importantly, the development of RV diastolic dysfunction occurred earlier in the Nrf2 knockout mice when compared to the wild type mice. SFN was able to attenuate RV dysfunctions in wild type PAH mice; however, SFN showed no protective effects in Nrf2 knockout PAH mice as measured by multiple RV and pulmonary parameters, including CI, PV VTI, TAPSE, RVSP, PAT/PET ratio, RVFW, RV E/E', dP/dT max, dP/dT min, and RV Tei index.

$\mathrm{Nrf} 2$ is a basic leucine zipper transcription factor that regulates redox balance and stress response through 

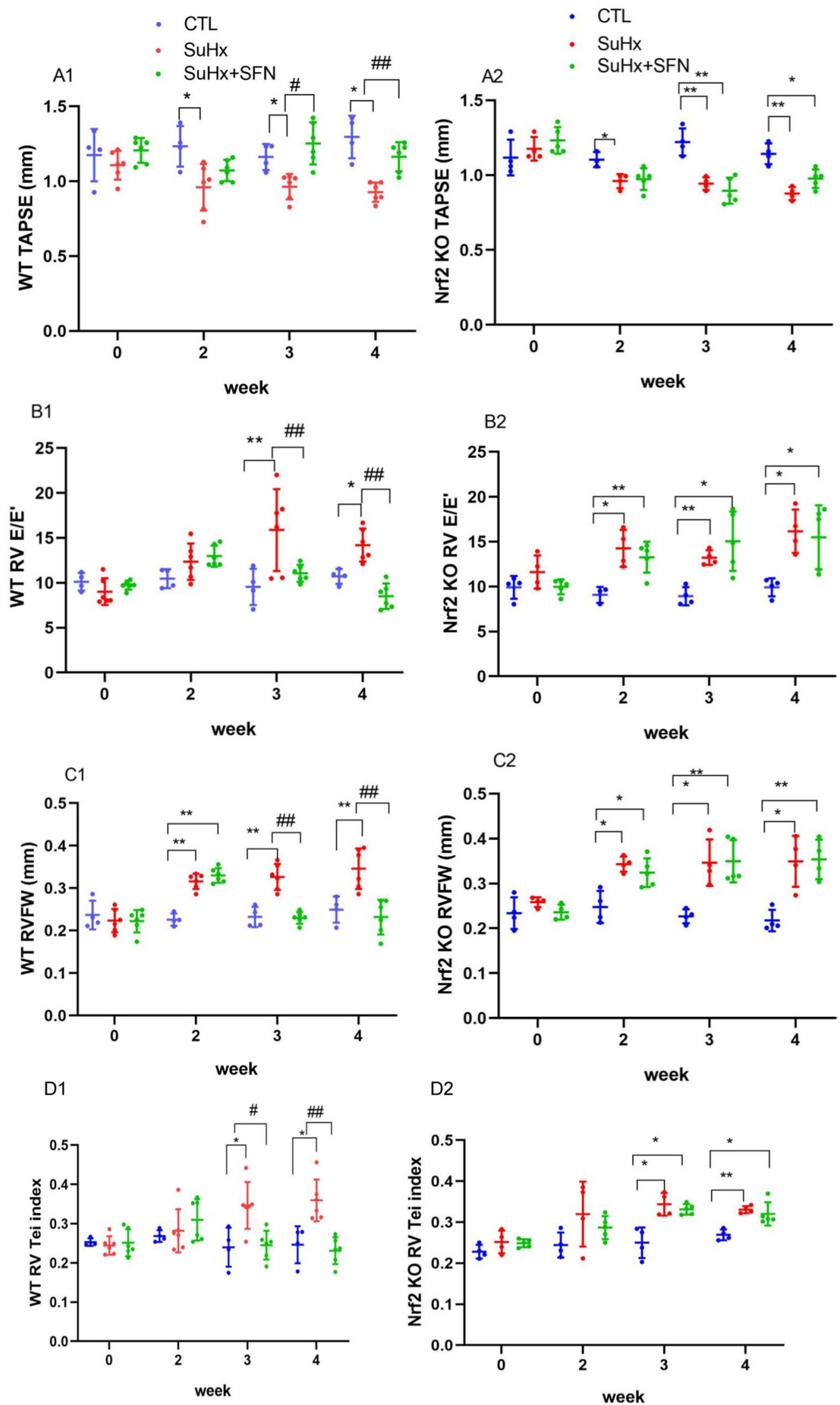
4Fig. 3 Increased RV free wall thickness (RVFW) in SuHx group was restored by SFN in wild type mice but not in Nrf2 knockout mice. Reduced RV diastolic function (E/E') and tricuspid annular systolic excursion with $\mathrm{SuHx}$ was attenuated by SFN in wild type mice but not in Nrf2 knockout mice. A1: RVFW, B1: E/E' ratio. C1: TAPSE in wild type mice. D1: RV Tei Index. $n=4,6,6$ for CTL (blue), SuHx (red), and $\mathrm{SuHx}+\mathrm{SFN}$ (green) groups, respectively. A2: RVFW, B2: E/E' ratio. C2: TAPSE in Nrf2 knockout mice. D1: RV Tei Index. $\mathrm{n}=4,4,5$ for CTL (blue), $\mathrm{SuHx}$ (red), and $\mathrm{SuHx}+\mathrm{SFN}$ (green) groups, respectively. $* P<0.05, * * P<0.01, \# P<0.05, \# \# P<0.01$ between indicated groups at the same time point

controlling the basal and inducible expression of a battery of antioxidant and other cytoprotective genes by binding to the antioxidant response element [16]. Under normal conditions, nuclear levels of Nrf2 are low; however, nuclear accumulation of Nrf2 increases under stress, resulting in enhanced transcriptional activation of its targets, and confers protection against various environmental stresses [17]. Disruption of Nrf2 dramatically increased the mortality of mice in response to septic shock with augmented expression of proinflammatory genes associated with the innate immune response in lungs of Nrf2deficient mice, indicating severe immune dysregulation [16]. Nrf2 was found to be the key regulator for the two important cytoprotective pathways, anti-inflammation and anti-oxidation, in murine macrophages [18]. In addition, Nrf2 improved lupus nephritis by neutralizing reactive oxygen species and by negatively regulating the $\mathrm{NF}-\kappa \mathrm{B}$ and TGF $\beta 1$ signaling pathways [19]. Our PAH mouse model demonstrated that Nrf2 deficiency accelerated the development of RV diastolic dysfunction in PAH mice; however, no significant difference was found in echocardiographic measurements of RV systolic function and invasive pulmonary pressures. We previously showed that hypoxia and Su5416 induced PAH and significant RV diastolic and systolic dysfunction along with markers of RV and pulmonary inflammation, fibrosis, and oxidative stress [15]. Evidence revealed that multiple inflammatory cells and pathways are activated in lungs of PAH patients [20] and also increased in the RV of animal PAH models [21]. The lack of worse or earlier RV systolic dysfunction in Nrf2 deficient mice could possibly be for several reasons: (1) SuHx effects on PAH and RV were so overwhelming that echocardiographic measurements at 2 weeks were not able to detect any difference between wild type and Nrf2 knockout mice. (2) SuHx induced PAH and RV dysfunction via Nrf2 independent pathways. (3) Nrf2 pathway might only be important in the development of RV dysfunction in late PAH pathogenesis. Interestingly, compared to WT mice, SFN exerted less protection in Nrf2 KO mice for CI, PV VTI, PAT/PET, TAPSE, RVFW, and RV Tei index at week 3, but only demonstrated less protection for CI, RV E/E',
RVFW, and RV Tei index at week 4. PV VTI, PAT/PET, and TAPSE were attenuated similarly in Nrf2 $\mathrm{KO}$ mice as WT mice at week 4 . This could reflect later activations of other anti-inflammatory, anti-oxidative genes by 4 weeks' administration of SFN via an Nrf2 independent pathway. Longer follow-ups to explore whether prolonged SFN administration could indeed confer more RV protections in Nrf2 KO mice and through which signaling pathways are critical.

Recently, two Nrf2 independent pathways have been implied in PAH and RV pathogenesis. Junctophilins (JP) protein plays roles in excitation-contraction coupling, such as promoting and stabilizing junctional membrane complexes, maintaining intracellular $\mathrm{Ca} 2+$ homeostasis, and being a transcription factor under stress [34]. JP cleavage inhibitor was shown to mitigate the severity of RV cardiomyocyte contractile dysfunction and attenuated right heart failure in acute PAH. In addition, a single cell sequencing study of pulmonary arterial endothelial cells isolated from PAH patients revealed significantly increased levels of metallothionein genes in PAH pulmonary arterial endothelial cells when compared to healthy controls [35], suggesting a possible role. These Nrf2 independent pathways might explain why Nrf2 KO mice did not develop a more accentuated PAH progression with SuHx and why SFN could still confer some protections of the RV after 4 weeks' administration in Nrf2 KO mice.

Preventing inflammation is important in various diseases, such as metabolic syndromes, autoimmune diseases, cancer, and chronic lung diseases [13, 22, 23]. Nrf2 activators have recently attracted interest to treat inflammatory diseases [24-26]. Bardoxolone, an activator of Nrf2, improved 6 min walking distances in PAH patients of the LARIAT trial (ClinicalTrials. gov Identifier: NCT02036970) [25]. Another Nrf2 activator, CXA-10, is hypothesized to be disease modifying by improving both pulmonary vasculature pathological remodeling as well as RV cardiac remodeling and dysfunction in patients with PAH. The PRIMEx study is investigating the safety and disease modifying effects of CXA-10 for the treatment of PAH (Clini calTrials.gov Identifier: NCT04053543). SFN is an isothiocyanate and potent Nrf2 activator and activates anti-inflammatory pathways related to Nrf2 activity, including protective effects on the left ventricle in diabetic cardiomyopathy and angiotensin II-induced cardiomyopathy $[27,28]$. Our previous study showed SFN could restore SuHx induced reduction of RV Nrf2 and downstream NQO1 expression, and impaired RV function and remodeling [15]. Our current results suggested that SFN may depend on the Nrf2 pathway to prevent PAH and RV dysfunction in the mouse PAH 


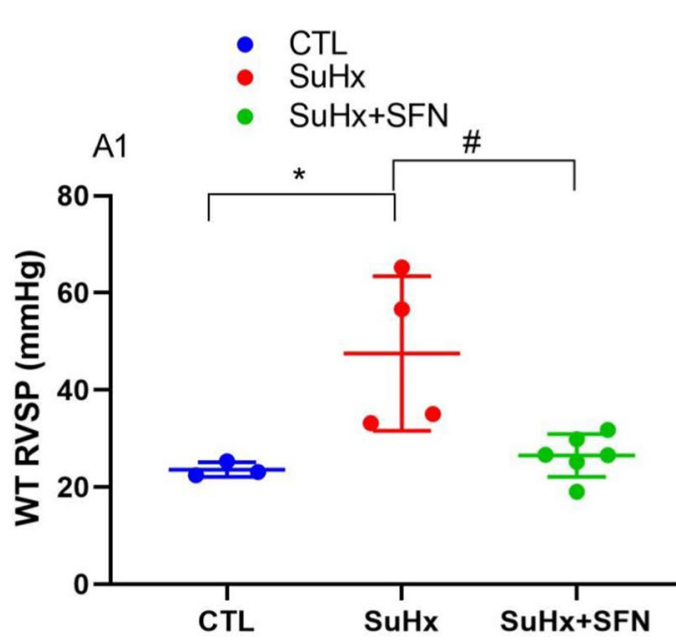

- CTL

- SuHx

A2 2 SuHx+SFN
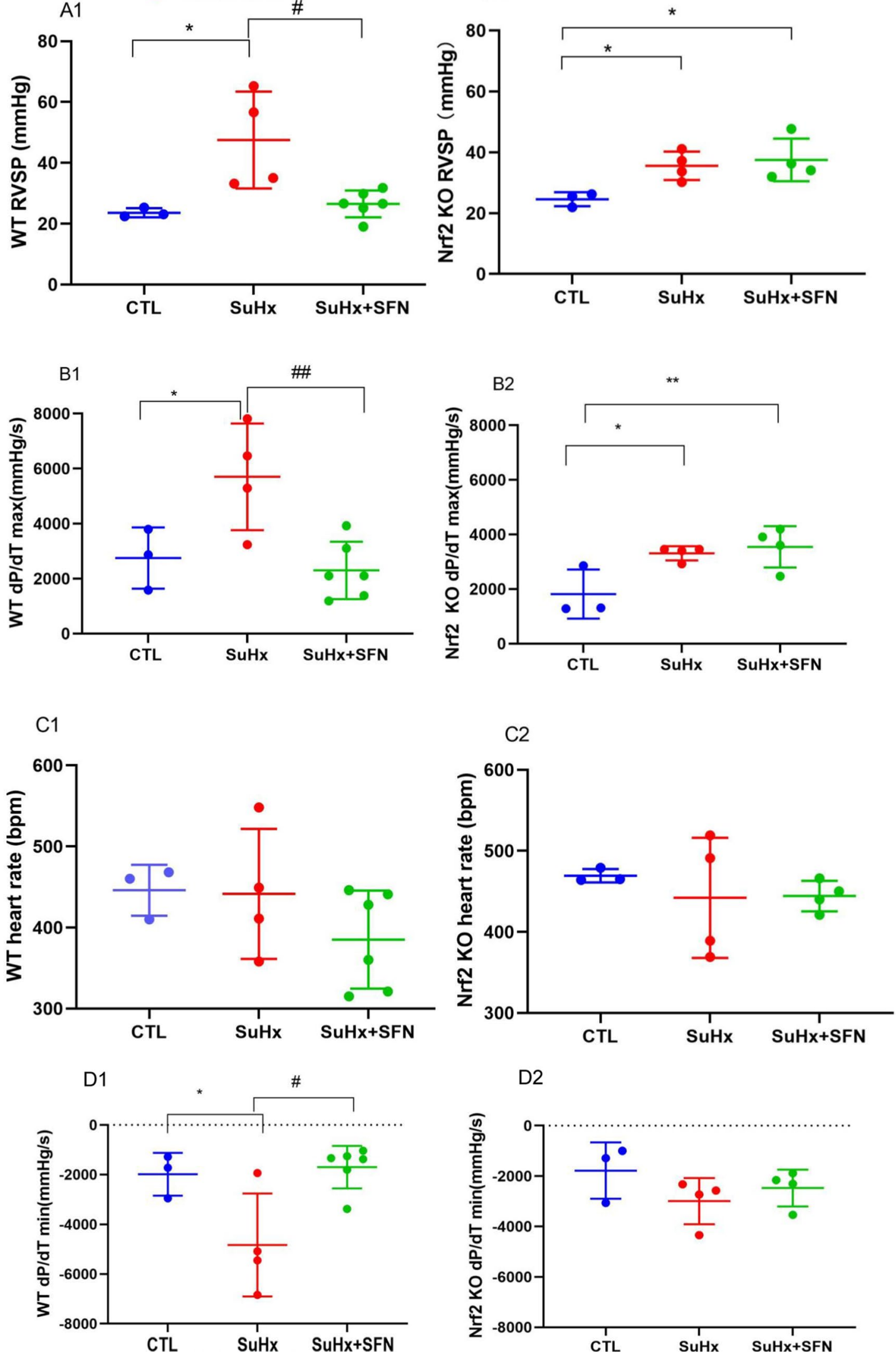

D2

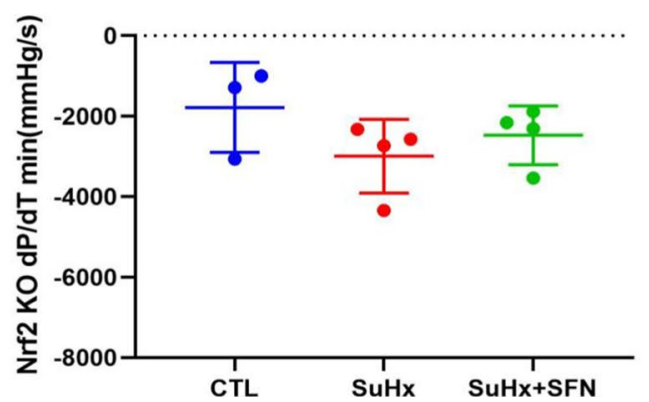


4Fig. 4 Increased RV peak systolic pressure by SuHx was prevented by SFN in wild type mice, but not in Nrf2 knockout mice. Heart rate was similar in all groups. A1: RV peak systolic pressure $(\mathrm{mmHg})$. B1: RV dP/dT max (mmHg/s). C1: Heart rate (bpm). D1: RV dP/dT $\min (\mathrm{mmHg} / \mathrm{s})$ in wild type mice. $n=3,4,6$ for CTL (blue), SuHx (red), and $\mathrm{SuHx}+\mathrm{SFN}$ (green) groups, respectively. A2: RV peak systolic pressure $(\mathrm{mmHg}), \mathrm{B} 2: \mathrm{RV} \mathrm{dP} / \mathrm{dT} \max (\mathrm{mmHg} / \mathrm{s})$. C2: Heart rate (bpm). D2: RV dP/dT min (mmHg/s) in Nrf2 knockout mice, $\mathrm{n}=3$, 4, 4 for CTL (blue), SuHx (red), and $\mathrm{SuHx}+\mathrm{SFN}$ (green) groups, respectively. $* P<0.05, \# P<0.05$ between indicated groups model based on the lack of protective effects of SFN in Nrf2 deficient PAH mice. We thus demonstrated the importance of the Nrf2 pathway in SFN mediated PAH and RV improvement in a mouse model through a loss of function method. SFN was given at $0.5 \mathrm{mg} / \mathrm{kg}$ s.c. for 5 days of each week as previously described from our laboratory [29]. To study different dosage of SFN and whether SFN can attenuate PH after it was induced is of

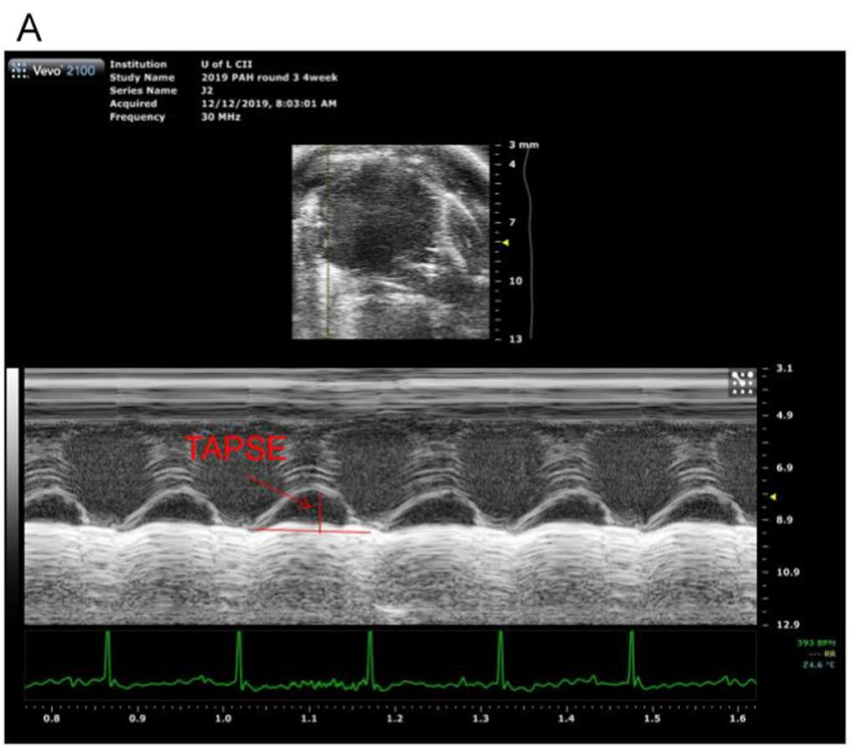

Representative waveform of TAPSE $\mathrm{B}$
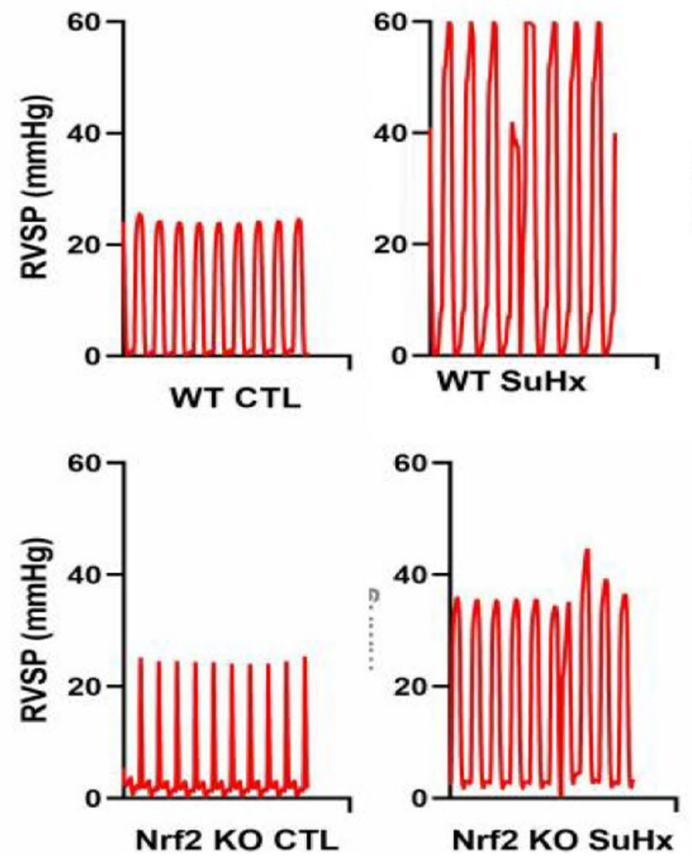

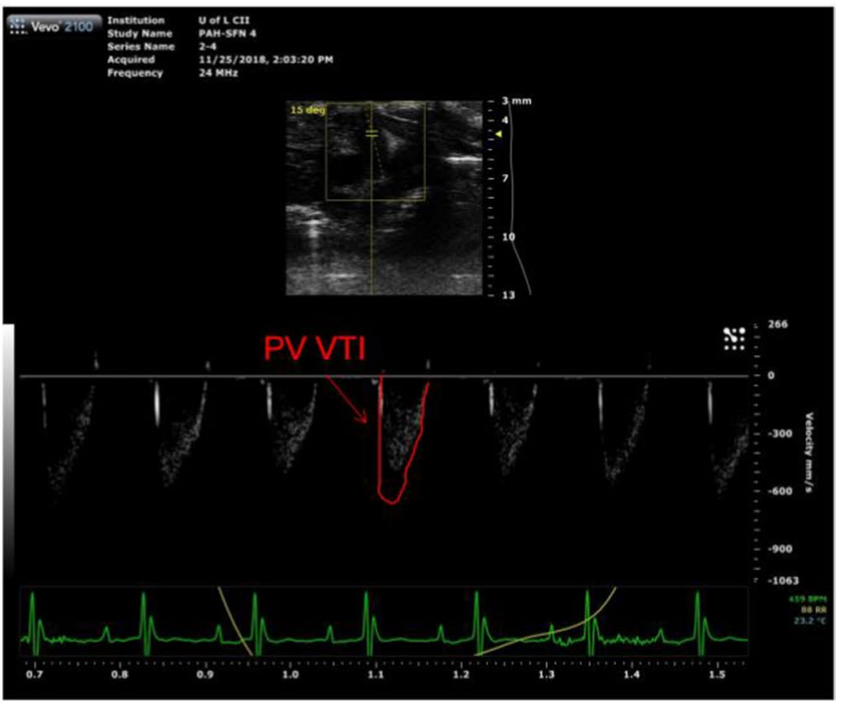

Representative pulmonary artery outflow
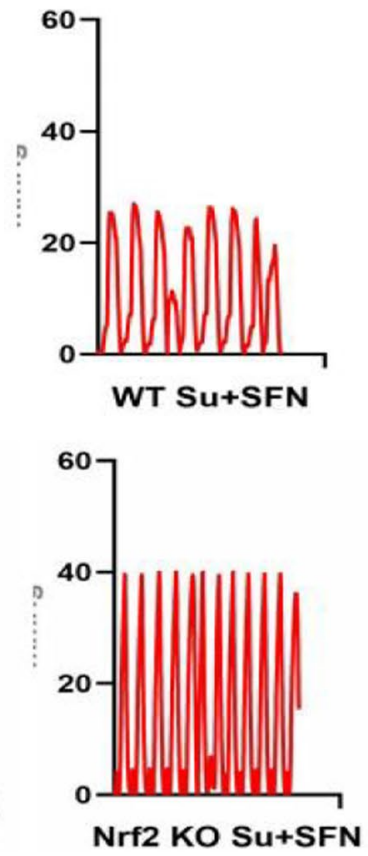

Fig. 5 A: Representative echocardiographic measurements for TAPSE and PV VTI. B: Waveform diagrams of right ventricular systolic pressure in WT and Nrf2 KO mice 
Table 1 Comparisons of echocardiographic variables between WT and $\mathrm{Nrf2}-\mathrm{KO}$ mice at week 2

\begin{tabular}{llllll}
\hline Variables & \multicolumn{2}{l}{ Ratio of SuHx to control } & \multicolumn{2}{l}{$\begin{array}{l}\text { Ratio of SuHx }+ \text { SFN to } \\
\text { SuHx }\end{array}$} \\
\cline { 2 - 3 } \cline { 5 - 6 } \cline { 5 - 6 } & WT & Nrf2-KO & & WT & Nrf2-KO \\
\hline CI & $0.78 \pm 0.13$ & $0.75 \pm 0.10$ & & $1.37 \pm 0.25$ & $0.81 \pm 0.09 *$ \\
PV VTI(mm) & $0.83 \pm 0.07$ & $0.93 \pm 0.08$ & & $1.11 \pm 0.25$ & $0.82 \pm 0.03$ \\
PAT/PET & $0.83 \pm 0.09$ & $0.82 \pm 0.04$ & & $1.08 \pm 0.14$ & $0.95 \pm 0.05$ \\
TAPSE(mm) & $0.83 \pm 0.09$ & $0.82 \pm 0.04$ & & $1.08 \pm 0.14$ & $0.95 \pm 0.15$ \\
RV E/E' & $1.28 \pm 0.30$ & $1.59 \pm 0.21$ & & $1.05 \pm 0.15$ & $1.00 \pm 0.15$ \\
RVFW & $1.39 \pm 0.17$ & $1.41 \pm 0.25$ & & $1.00 \pm 0.07$ & $0.94 \pm 0.08$ \\
RV Tei Index & $0.96 \pm 0.09$ & $1.34 \pm 0.34$ & & $1.14 \pm 0.31$ & $0.93 \pm 0.17$ \\
\hline
\end{tabular}

${ }^{*} P<0.05 \mathrm{Nrf} 2-\mathrm{KO}$ vesus WT
SFN protection in PAH. Third, we performed echocardiographic measurements at week 2 after PAH has been initiated. Earlier echocardiographic measurements might reveal additional important differences between wild type and Nrf2 knockout mice. Fourth, we only studied Nrf2 knockout mice in one specific PAH model, SuHx. Whether these effects are reproducible in other PAH models or humans are unknown.

In summary, our present study provides evidence that SFN is dependent upon the Nrf2 pathway to prevent RV dysfunction and reduce adverse pulmonary vascular remodeling in the SuHx-induced PAH mouse model. Preclinical and clinical studies are needed to study if the induction of the Nrf2 pathway could improve RV function and reduce $\mathrm{PAH}$ in patients.
Table 2 Comparisons of echocardiographic variables between WT and Nrf2-KO mice at week 3

Table 3 Comparisons of echocardiographic variables between WT and Nrf2-KO mice at week 4

\begin{tabular}{|c|c|c|c|c|}
\hline \multirow[t]{2}{*}{ Variables } & \multicolumn{2}{|c|}{ Ratio of SuHx to control } & \multicolumn{2}{|c|}{ Ratio of $\mathrm{SuHx}+\mathrm{SFN}$ to $\mathrm{SuHx}$} \\
\hline & WT & Nrf2-KO & WT & Nrf2-KO \\
\hline CI & $0.77 \pm 0.13$ & $0.74 \pm 0.10$ & $1.33 \pm 0.24$ & $0.90 \pm 0.15^{*}$ \\
\hline PV VTI(mm) & $0.86 \pm 0.05$ & $0.81 \pm 0.04$ & $1.18 \pm 0.10$ & $0.99 \pm 0.09^{*}$ \\
\hline PAT/PET & $0.78 \pm 0.05$ & $0.77 \pm 0.09$ & $1.38 \pm 0.09$ & $1.05 \pm 0.06^{* * *}$ \\
\hline TAPSE(mm) & $0.78 \pm 0.05$ & $0.77 \pm 0.09$ & $1.38 \pm 0.09$ & $1.049 \pm 0.06^{* * *}$ \\
\hline RV E/E' & $1.72 \pm 0.65$ & $1.50 \pm 0.23$ & $0.76 \pm 0.29$ & $1.19 \pm 0.31$ \\
\hline RVFW & $1.54 \pm 0.31$ & $1.54 \pm 0.27$ & $0.70 \pm 0.16$ & $0.97 \pm 0.05^{*}$ \\
\hline RV Tei Index & $1.45 \pm 0.07$ & $1.40 \pm 0.20$ & $0.72 \pm 0.17$ & $0.96 \pm 0.08 *$ \\
\hline
\end{tabular}

$* P<0.05, * * P<0.01, * * * P<0.005 \mathrm{Nrf} 2-\mathrm{KO}$ vesus $\mathrm{WT}$

\begin{tabular}{|c|c|c|c|c|}
\hline \multirow[t]{2}{*}{ Variables } & \multicolumn{2}{|c|}{ Ratio of SuHx to control } & \multicolumn{2}{|c|}{ Ratio of $\mathrm{SuHx}+\mathrm{SFN}$ to $\mathrm{SuHx}$} \\
\hline & WT & Nrf2-KO & WT & Nrf2-KO \\
\hline CI & $0.79 \pm 0.20$ & $0.67 \pm 0.19$ & $1.48 \pm .12$ & $0.92 \pm 0.13^{* * *}$ \\
\hline PV VTI(mm) & $0.79 \pm 0.06$ & $0.82 \pm 0.04$ & $1.17 \pm 0.11$ & $1.04 \pm 0.13$ \\
\hline PAT/PET & $0.71 \pm 0.01$ & $0.77 \pm 0.01$ & $1.28 \pm 0.02$ & $1.02 \pm 0.26$ \\
\hline TAPSE(mm) & $0.71 \pm 0.11$ & $0.77 \pm 0.11$ & $1.28 \pm 0.19$ & $1.023 \pm 0.26$ \\
\hline RV E/E' & $1.35 \pm 0.28$ & $1.65 \pm 0.35$ & $0.60 \pm 0.11$ & $1.053 \pm 0.34^{*}$ \\
\hline RVFW & $1.51 \pm 0.34$ & $1.61 \pm 0.22$ & $0.63 \pm 0.18$ & $1.10 \pm 0.16^{*}$ \\
\hline RV Tei Index & $1.45 \pm 0.32$ & $1.23 \pm 0.07$ & $0.66 \pm 0.16$ & $0.93 \pm 0.01 *$ \\
\hline
\end{tabular}

$* P<0.05, * * * P<0.005$ Nrf $2-\mathrm{KO}$ vesus WT great importance and we plan to perform these experiments in the future.

Our current study has several limitations. First, we only used male Nrf2 knockout mice in this experiment. We plan to include female mice in a future study to investigate the gender differences. Second, we only performed echocardiographic RV function assessments and invasive RV systolic pressure calculations in this experiment. We plan to explore histological and molecular markers in Nrf2 knockout PAH mice with and without SFN to elucidate the mechanisms of
Authors' Contributions $\mathrm{YZ}$ and $\mathrm{YK}$ designed experiments, performed experiments, interpreted data, and prepared the manuscript. DC and AJL performed supplemental experiments. AJL, JC, LC, SW, JH, and $\mathrm{BBK}$ designed the experiments, interpreted data, and revised the manuscript.

Funding This work was supported in part by the University of Louisville-China Pediatric Research Exchange Program (LC, JC, JH, and BBK, no salary support), University of Louisville Executive Vice President for Research and Innovation Internal Grant (JH, LC), University of Louisville School of Medicine Basic Grant (JH, LC), National Institute of Environmental Health Sciences (P30ES030283 to LC, JC and 
JH), Gilead Sciences COMMIT COVID-19 RFP Program grant (Gilead IN-US-983-6063 to JH), National Center for Advancing Translational Sciences grant (1U18TR003787-01 support for JH), National Institute on Aging of the National Institutes of Health (R44AG060848 support for JH), and by the National Institute of Nursing Research of the National Institutes of Health (R43NR017372 support for JH), Natural Science Foundation Grant from the Guangdong Province of China (No: 2018A03031353 to SW), and Medical Scientific Research Foundation Grant of Guangdong Province of China (No: A2021338 to YK). All personnel expenses and partial research-related expenses for Dr. Yin Kang and Dr. Guangyan Zhang were provided by the Department of Anesthesiology, Guangdong Provincial People's Hospital, Guangdong Academy of Medical Sciences, Guangzhou, China. All basic science experiments were completed at the University of Louisville, Louisville, KY, USA.

Availability of Data and Materials Available upon request

\section{Declarations}

Ethics Approval and Consent to Participate Not applicable.

Consent for Publication Not applicable.

Competing Interests The authors declare that they have no competing interests.

Ethics Approval and Consent to Participate Not applicable.

Research Involving Human Participants and/or Animals All experimental procedures were approved by the Institutional Animal Care and Use Committee of the University of Louisville.

Informed Consent Not applicable.

\section{References}

1. Ryan JJ, Archer SL. The right ventricle in pulmonary arterial hypertension: disorders of metabolism, angiogenesis and adrenergic signaling in right ventricular failure. Circ Res. 2014;115(1):176-88.

2. Galiè N, Humbert M, Vachiery JL, et al. 2015 ESC/ERS guidelines for the diagnosis and treatment of pulmonary hypertension. Rev Esp Cardiol (Engl Ed). 2016;69(2):177.

3. Kovacs G, Dumitrescu D, Barner A, et al. Definition, clinical classification and initial diagnosis of pulmonary hypertension: updated recommendations from the Cologne consensus conference 2018. Int J Cardiol. 2018;272S:11-9.

4. Vonk-Noordegraaf A, Haddad F, Chin KM, et al. Right heart adaptation to pulmonary arterial hypertension: physiology and pathobiology. J Am Coll Cardiol. 2013;62(25 Suppl):D22-33.

5. Xiao Y, Chen PP, Zhou RL, Zhang Y, Tian Z, Zhang SY. Pathological mechanisms and potential therapeutic targets of pulmonary arterial hypertension: a review. Aging Dis. 2020;11(6):1623-39.

6. Pugliese SC, Poth JM, Fini MA, Olschewski A, El Kasmi KC, Stenmark KR. The role of inflammation in hypoxic pulmonary hypertension: from cellular mechanisms to clinical phenotypes. Am J Physiol Lung Cell Mol Physiol. 2015;308(3):L229-52.

7. Kuebler WM, Bonnet S, Tabuchi A. Inflammation and autoimmunity in pulmonary hypertension: is there a role for endothelial adhesion molecules? (2017 Grover conference series). Pulm Circ. 2018;8(2):2045893218757596.
8. Wang JJ, Zuo XR, Xu J, et al. Evaluation and treatment of endoplasmic reticulum (ER) stress in right ventricular dysfunction during Monocrotaline-induced rat pulmonary arterial hypertension. Cardiovasc Drugs Ther. 2016;30(6):587-98.

9. Dewachter C, Belhaj A, Rondelet B, et al. Myocardial inflammation in experimental acute right ventricular failure: effects of prostacyclin therapy. J Heart Lung Transplant. 2015;34(10):1334-45.

10. Fontoura D, Oliveira-Pinto J, Tavares-Silva M, et al. Myocardial and anti-inflammatory effects of chronic bosentan therapy in monocrotaline-induced pulmonary hypertension. Rev Port Cardiol. 2014;33(4):213-22.

11. Van Tassell BW, Abouzaki NA, Oddi Erdle C, et al. Interleukin-1 blockade in acute decompensated heart failure: a randomized, double-blinded. Placebo-Controlled Pilot Study J Cardiovasc Pharmacol. 2016;67(6):544-51.

12. Shintani Y, Maruoka S, Gon Y, et al. Nuclear factor erythroid 2-related factor 2 (Nrf2) regulates airway epithelial barrier integrity. Allergol Int. 2015;64(Suppl):S54-63.

13. Barnes PJ. Inflammatory mechanisms in patients with chronic obstructive pulmonary disease. J Allergy Clin Immunol. 2016;138(1):16-27.

14. Wang Q, Ma J, Lu Y, et al. CDK20 interacts with KEAP1 to activate NRF2 and promotes radiochemoresistance in lung cancer cells. Oncogene. 2017;36(37):5321-30.

15. Kang Y, Zhang G, Huang EC, et al. Sulforaphane prevents right ventricular injury and reduces pulmonary vascular remodeling in pulmonary arterial hypertension. Am J Physiol Heart Circ Physiol. 2020;318(4):H853-66.

16. Thimmulappa RK, Lee H, Rangasamy T, et al. Nrf2 is a critical regulator of the innate immune response and survival during experimental sepsis. J Clin Invest. 2006;116(4):984-95.

17. Rangasamy T, Cho CY, Thimmulappa RK, et al. Genetic ablation of Nrf2 enhances susceptibility to cigarette smoke-induced emphysema in mice. J Clin Invest. 2004;114(9):1248-59.

18. Kobayashi EH, Suzuki T, Funayama R, et al. Nrf2 suppresses macrophage inflammatory response by blocking proinflammatory cytokine transcription. Nat Commun. 2016;7:11624.

19. Jiang T, Tian F, Zheng H, et al. Nrf2 suppresses lupus nephritis through inhibition of oxidative injury and the NF- $\mathrm{KB}$-mediated inflammatory response. Kidney Int. 2014;85(2):333-43.

20. Le Hiress M, Tu L, Ricard N, et al. Proinflammatory signature of the dysfunctional endothelium in pulmonary hypertension. Role of the macrophage migration inhibitory factor/CD74 complex. Am J Respir Crit Care Med. 2015;192(8):983-97.

21. Handoko ML, de Man FS, Happé CM, et al. Opposite effects of training in rats with stable and progressive pulmonary hypertension. Circulation. 2009;120(1):42-9.

22. Donath MY. Targeting inflammation in the treatment of type 2 diabetes: time to start. Nat Rev Drug Discov. 2014;13(6):465-76.

23. Fernandes JV, Cobucci RN, Jatobá CA, Fernandes TA, de Azevedo JW, de Araújo JM. The role of the mediators of inflammation in cancer development. Pathol Oncol Res. 2015;21(3):527-34.

24. Hensley MK, Levine A, Gladwin MT, Lai YC. Emerging therapeutics in pulmonary hypertension. Am J Physiol Lung Cell Mol Physiol. 2018;314(5):L769-81.

25. Sommer N, Ghofrani HA, Pak O, et al. Current and future treatments of pulmonary arterial hypertension. Br J Pharmacol. 2021;178(1):6-30.

26. Palliyaguru DL, Yuan JM, Kensler TW, Fahey JW. Isothiocyanates: translating the power of plants to people. Mol Nutr Food Res. 2018;62(18):e1700965.

27. Posner GH, Cho CG, Green JV, Zhang Y, Talalay P. Design and synthesis of bifunctional isothiocyanate analogs of sulforaphane: correlation between structure and potency as inducers of anticarcinogenic detoxication enzymes. J Med Chem. 1994;37(1):170-6. 
28. Fahey JW, Haristoy X, Dolan PM, et al. Sulforaphane inhibits extracellular, intracellular, and antibiotic-resistant strains of helicobacter pylori and prevents benzo[a]pyrene-induced stomach tumors. Proc Natl Acad Sci U S A. 2002;99(11):7610-5.

29. Gu J, Cheng Y, Wu H, et al. Metallothionein is downstream of Nrf2 and partially mediates Sulforaphane prevention of diabetic cardiomyopathy. Diabetes. 2017;66(2):529-42.

30. Xin Y, Bai Y, Jiang X, et al. Sulforaphane prevents angiotensin II-induced cardiomyopathy by activation of Nrf2 via stimulating the Akt/GSK-3ß/Fyn pathway. Redox Biol. 2018;15:405-17.

31. Zhou S, Wang J, Yin X, et al. Nrf2 expression and function, but not MT expression, is indispensable for sulforaphane-mediated protection against intermittent hypoxia-induced cardiomyopathy in mice. Redox Biol. 2018;19:11-21.

32. Leeuwenburgh BP, Steendijk P, Helbing WA, Baan J. Indexes of diastolic RV function: load dependence and changes after chronic RV pressure overload in lambs. Am J Physiol Heart Circ Physiol. 2002;282(4):H1350-8.
33. Stein PD, Sabbah HN, Mazilli M, Anbe DT. Effect of chronic pressure overload on the maximal rate of pressure fall of the right ventricle. Chest. 1980;78(1):10-5.

34. Tan R, Li C, Xu C, et al. Targeting JP2: a new treatment for pulmonary hypertension. Oxidative Med Cell Longev. 2021;2021:2003446.

35. Asosingh K, Comhair S, Mavrakis L, et al. Single-cell transcriptomic profile of human pulmonary artery endothelial cells in health and pulmonary arterial hypertension. Sci Rep. 2021;11(1):14714.

Publisher's Note Springer Nature remains neutral with regard to jurisdictional claims in published maps and institutional affiliations. 